\title{
Thin-film heterostructures based on conducting polymers and organic semiconductors
}

\author{
Ya.I. Vertsimakha ${ }^{1}$, O.I. Aksimentyeva ${ }^{2}$, R.J. Perminov ${ }^{1}$, D.O. Poliovyi ${ }^{2}$ \\ ${ }^{1}$ Institute of Physics, NAS of Ukraine, 46, prospect Nauky, 03028 Kyiv, Ukraine, \\ Phone: (380-044)-525-09-12,e-mail: yavertsi@iop.kiev.ua \\ ${ }^{2}$ I. Franko L'viv National University, 6, Kyryla-Mefodia Str., Lviv 79005, Ukraine
}

\begin{abstract}
We have carried out the experimental verification of a possibility to produce organic heterostructures by using films of organic semiconductors (OS) which are photosensitive in a wide spectral region, absorb light, and generate charge carriers in the region of transparency of films of conducting polymers - electrochemically synthesized polyaniline (PAN) and poly-3,4-ethylenedioxythiophene (PEDOT) stabilized by polystyrenesulfone acid (PSS). As components for the fabrication of these heterostuctures, we chose photosensitive organic semiconductors of the $n$-type, $N, N$ dimethyl-3,4,9,10-perylenetetracarboxydiimide (MPP), and of the $p$-type, pentacene $(\mathrm{Pn})$, which were thermally sprayed on thin layers of PAN and PEDOT. The photovoltage of heterostuctures produced at the temperature of substrates $370 \mathrm{~K}$ is several times greater than that of components, reaches its maximum in the region of strong light absorption in OS layers, but is one order less in the region of strong absorption in polymers. This testifies to the appearance of an internal electric field near the polymer/OS boundary which enhances the efficiency of separation of photogenerated charge carriers, whereas the efficiency inherent to photogeneration of carriers in the layers with PAN and PEDOT-PSS is insufficient yet.
\end{abstract}

Keywords: heterostructure, conducting polymer, organic semiconductor, photovoltaic effect.

Manuscript received 17.02.09; revised manuscript received 18.03.09; accepted for publication 14.05.09; published online 15.05.09.

\section{Introduction}

Efficient absorption of solar light, which leads to the photogeneration of charge carriers, is a necessary condition for the choice of components for solar cells. Recently, a great attention was paid to organic solar cells that are rather technological, and the application of polymeric film components allows one to obtain flexible (pliable) solar cells [1-3]. At the same time, there appears a number of difficulties on the development of pliable solar cells, which is related, first of all, to a low efficiency of the photogeneration of charge carriers in polymers. In addition, the process of charge separation in these systems at the polymer-electrode boundary has a low efficiency, which is caused by a large density of uncontrolled impurities acting as traps for excitons and charge carriers [1]. The organic solar cells developed earlier on the basis of conducting polymers and derivatives of $\mathrm{C}_{60}$ absorb only a part of solar light in the region $400-700 \mathrm{~nm} \mathrm{[1],} \mathrm{whereas} \mathrm{the} \mathrm{composites}$ produced from carbazole-containing polymers and polymethine dyes absorb in the region of 550-950 nm
$[2,3]$. Therefore, the important task is the search for ways to extend the range of photosensitivity of organic heterostuctures to the long-wave or/and short-wave regions.

The most attractive by their physico-chemical and electrophysical properties, simple in synthesis, and possessing sufficiently high stability are such conducting polymers as electrochemically synthesized polyaniline (PAN) and poly-3,4-ethylenedioxythiophene (PEDOT).

The PAN films on a solid surface can be produced by both the electrochemical method [4] and that of thermal vacuum spraying [5]. The properties of films produced by these methods are very different. An advantage of PAN films produced by the electrochemical method is their efficient absorption in the near-IR region, whereas the two-layer structures of PAN with films of many organic semiconductors (OS) absorb solar light in a wide spectral region (400$1400 \mathrm{~nm})$. As a drawback, we indicate a rather low photosensitivity of PAN films themselves, which is probably caused by structural peculiarities of polyaminoarenes, a high level of intrinsic conductivity, 
and the contribution of ion conductivity in electrochemically doped specimens. When keeping in air, the PAN films can adsorb gaseous impurities, volatile components of solvents, and polar gases, which increases the possibility for charge carriers to recombine at adsorption centers. Because the binding energy of these adsorbed impurities with macromolecules is low, it is probable that the number of surface adsorption centers can be significantly decreased by annealing, especially in vacuum.

Therefore, the aim of our work was both the experimental verification of the photosensitivity of heterostructures of PAN and PEDOT with OS films, which absorb light and generate charge carriers in the region of weak absorption of polymeric films, and the choice of an optimal structure for the development of solar cells.

\section{Technology and method of the experiment}

The thin PAN films were deposited on the purified and degreased surface of ITO-electrodes by the electrochemical polymerization of a $0.1 \mathrm{M}$ aniline solution in $0.5 \mathrm{M}$ sulfate acid at $T=293 \mathrm{~K}$. The synthesis was performed in a quartz cell with special structure, and the operation electrode was of a disk-like shape of 12 or $16 \mathrm{~mm}$ in diameter and was fixed by $\mathrm{Pt}$ holders. As a counterelectrode, we used a Pt wire, and the reference electrode was made of $\mathrm{Ag} / \mathrm{AgCl}$. The current density was $0.05-0.1 \mathrm{~mA} / \mathrm{cm}^{2}$ to provide the potential of the operation electrode $E=0.78-0.82 \mathrm{~V}$. The cell was placed to the operation chamber of a spectrophotometer CF-46 and connected with a potentiostat PI-50-M1. The electrolysis duration was varied within the limits of 10-15 min. The thickness of the obtained PAN layer after the application was controlled by the optical density $(D)$ of the film at the wavelength $\lambda=750 \mathrm{~nm}$ with an interference metallomicroscope MII-4.

A 1.3-wt.\% aqueous dispersion of conducting polymer PEDOT doped with polystyrenesulfone acid (PSS) was purchased from Aldrich. Five milliliters of a PEDOT-PSS aqueous dispersion was mixed with $120 \mu \mathrm{l}$ glycerin, $250 \mu \mathrm{l} \quad \mathrm{N}$-methyl-2-pyrrolidone (Riedelde Haën), $6.25 \mathrm{ml}$ isopropanol, and $81 \mu \mathrm{l}$ tetraethoxysilane (Fluka) to prepare highly conductive mechanically stable coatings with the surface resistivity below $10^{3} \mathrm{Ohm} / \square$. A Chemat Technology KW-4A precision spin-coater was used to deposit polymer coatings. The rotation speeds 800 r.p.m. for $9 \mathrm{~s}$ and of 3000 r.p.m. for $30 \mathrm{~s}$ were applied to produce high-quality smooth PEDOT-PSS films with the thickness close to $50 \mathrm{~nm}$. The mixture was spin-coated on CIS substrates and dried at $310 \mathrm{~K}$ for $24 \mathrm{~h}$. After evaporation of solvents, the obtained structures were annealed at $460 \mathrm{~K}$ for $4 \mathrm{~min}$. To prepare golden contact grids, gold was evaporated in vacuum on the surface of PEDOT-PSS films.
As components to produce photosensitive heterostructures, we took organic semiconductors $N, N$ dimethyl-3,4,9,10-perylenetetracarboxydiimide (MPP) of the $n$-type [6] and pentacene (Pn) of the $p$-type [7, 8], which were thermally sprayed on layers of PAN at the substrate temperature $\left(T_{s}\right) 370 \mathrm{~K}$.

The surface topology of the obtained PAN and OS film specimens was studied using an atomic-force microscope (AFM) "NanoScope IIIa". The software of the PSI firm allowed us to register and process the data, to perform the filtration and smoothing, and to carry out the statistical analysis of the obtained patterns.

The heterostructures were fabricated by means of a subsequent thermal spraying of layers of Pn or MPP in vacuum on substrates at various $T_{\mathrm{s}}$. The thickness of OS layers was controlled during the process of spraying by a change in the frequency of a quartz resonator and, after their fabrication, by spectra of the optical density and using AFM. The thickness of OS layers was close to $50 \mathrm{~nm}$. The absorption spectra were obtained using a two-beam spectrophotometer „Hitachi” or a spectrophotometer SA-46 at $T=293 \mathrm{~K}$.

The measurement of photovoltage was performed by the non-contact capacitance Bergman method with modulated illumination $[6,9]$. The monochromatic light power was measured by a calibrated radiometer PPTN02 based on a Si photodiode.

\section{Experimental results}

\subsection{Morphology of films of polyaniline and organic semiconductors}

The studies of the surface structure of an electrochemically obtained Pn film by using AFM showed that PAN films are mainly fine-crystalline prior to the annealing (the size of crystallites was approximately $100 \mathrm{~nm}$ ) with a small number of aggregates on the surface of $0.5-1.0 \mu \mathrm{m}$ in size - the maximal values of heights $Z=1.1 \mu \mathrm{m}$, and their rootmean-square deviation $r m s=136 \mathrm{~nm}$ (Fig. 1a). As a result of annealing, $Z, r m s$, and sizes of separate aggregates increased only by $\approx 20 \%$. The obtained values of $Z$ and rms were not larger than those in films of MPP $(Z=0.865 \mu \mathrm{m}, r m s=126 \mathrm{~nm})$ and Pn produced by thermal vacuum spraying (Fig. 1b) [6].

\subsection{Optical and photovoltaic properties of polymeric films}

The spectra of the films produced from components of the heterostuctures under study are presented in Fig. 2. It is seen that the two-layer polymer/OS structures absorb light well in the whole visible and near-IR spectral region (400$900 \mathrm{~nm}$ ). As an example, Fig. 3 (curve 2) gives the absorption spectrum of the PAN/Pn heterostructure. The difference of curves 2 and 1 corresponds to the absorption spectrum of Pn films (curve 3). By the ratio of the intensities for components of the Davydov doublet inherent to the first electron transition at 1.85 and $1.98 \mathrm{eV}$, 


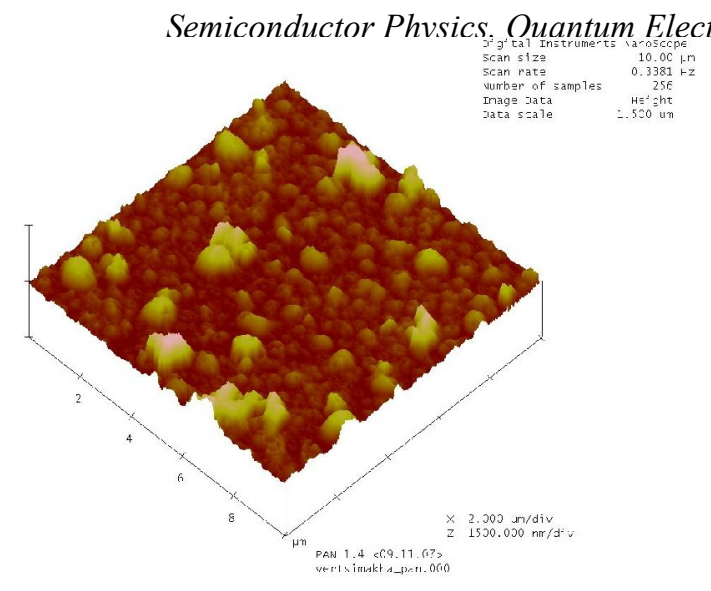

a

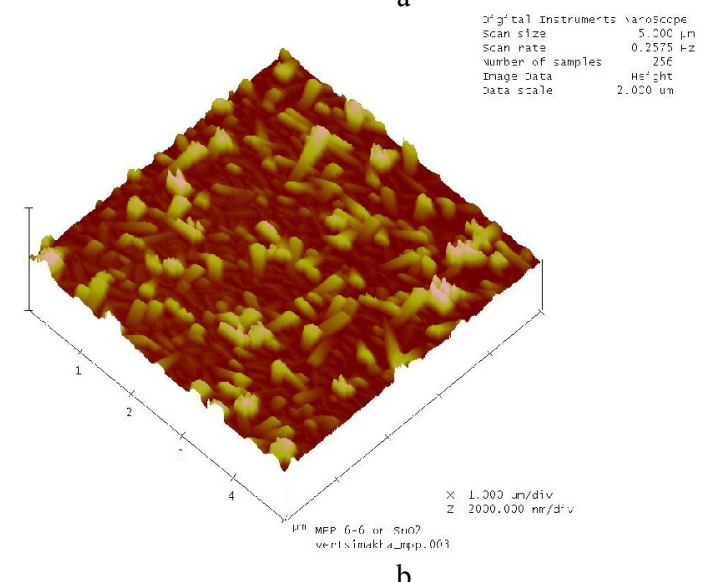

Fig. 1. AFM images of surface for polianiline films prepared by electrochemical method before annealing (a) and for MPP films thermally deposited at the substrate temperature $370 \mathrm{~K}(\mathrm{~b})$.

it is seen that an oriented polycrystalline Pn film is formed on the PAN film surface [10]. That is, the presence of a PAN film on the glass substrate does not practically affect the formation of Pn crystallites.

A similar pattern is observed for the PEDOT $+\mathrm{Pn}$, PAN+MPP, and PEDOT+MPP structures. The photovoltage $(\varphi)$ values of the studied films of PAN and PEDOT-PSS deposited on ITO layers on glass substrates are two orders less than those of MPP and PN layers thermally sprayed on similar substrates. Therefore, we failed to measure the spectral dependence of $\varphi$ for films of PAN and PEDOT-PSS using a monochromator, and the measurements of their $\varphi$ values were performed by the capacitance method under illumination with a modulated emission of light-emitting diodes with various quantum energies and from both sides. The studies showed that photovoltage values of the ITO/PAN structures are one order less under illumination of the free surface $\left(\varphi_{s}\right)$ than those under illumination through the ITO-electrode $\left(\varphi_{c}\right)$. Since there is no desorption of chemisorbed moisture from PAN films at these temperatures [5], the less values of $\varphi_{s}$ can be caused by the recombination of photogenerated charge carriers on defects of the surface (complexes) that arose due to the adsorption of gases and impurities present in air (oxygen, carbon dioxide, and others). However, after

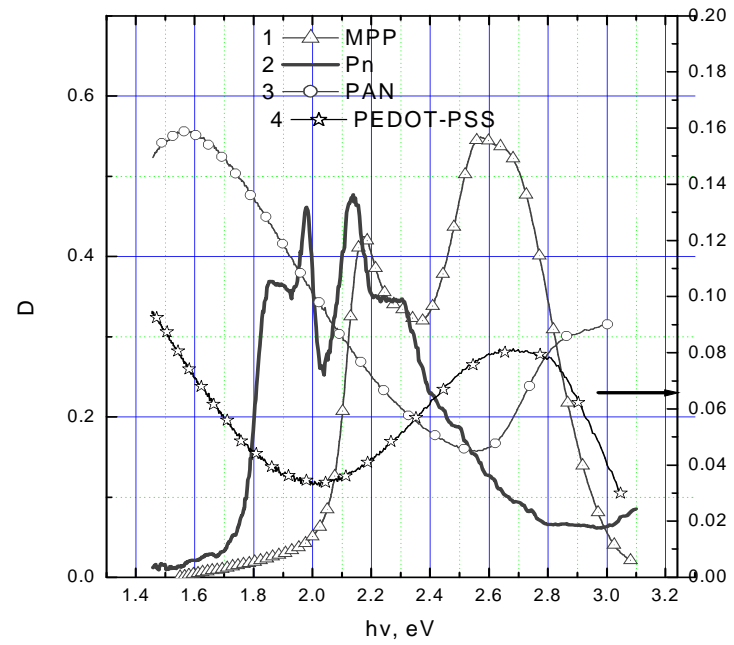

Fig. 2. Optical density spectra $D$ for components of heterostructures: MPP (1), Pn (2), PAN (3) and PEDOT-PSS (4).

annealing in air for $3 \mathrm{~h}$ at the temperature of $370 \mathrm{~K}\left(20^{\circ}\right.$ less than the temperature of PAN sublimation in vacuum [5]) with the subsequent gradual cooling down to room temperature, its $\varphi_{s}$ increases by a factor of 2.5 to 3 mainly in the region of weak absorption of PAN films (Table). Probably, this occurs due to desorption of impurities and the decay of surface complexes.

On the contrary, in the ITO/PEDOT-PSS structures $\varphi_{s}$ is almost one order higher than $\varphi_{c}$. In this case, it is probable that the adsorbed gases form centers, majority of which are centers providing photogeneration of charge carriers.

Table. The photovoltage of structures based on films PAN and PEDOT with films Pn and MPP under illumination of free surface of light-emitted diodes.

\begin{tabular}{|c|c|c|c|c|}
\hline $\begin{array}{c}\text { Illumination energy, eV } \\
\rightarrow\end{array}$ & 1.32 & 1.90 & 2.18 & 2.64 \\
\hline Structures $\downarrow$ & \multicolumn{4}{|c|}{ Photovoltage, V/W $\downarrow$} \\
\hline $\begin{array}{c}\text { ITO/PAN - before } \\
\text { annealing }\end{array}$ & 0.2 & 3 & 4 & 0.25 \\
\hline $\begin{array}{c}\text { ITO/PEDOT- before } \\
\text { annealing }\end{array}$ & 0.8 & 20 & 21 & 9 \\
\hline $\begin{array}{c}\text { ITO/PAN - after } \\
\text { annealing }\end{array}$ & 0.5 & 8 & 6 & 1.6 \\
\hline ITO/Pn & 0.2 & 1060 & 257 & 750 \\
\hline ITO/PAN/Pn & 0.4 & 2000 & 640 & 160 \\
\hline $\begin{array}{c}\text { ITO/PEDOT/Pn } \\
\text { ITO/MPP }\end{array}$ & 2 & 50000 & 2140 & 6430 \\
\hline ITO/PAN /MPP & 0.42 & 83 & 385 & 160 \\
\hline ITO/PEDOT/MPP & 8 & 470 & 4700 & 4820 \\
\hline
\end{tabular}




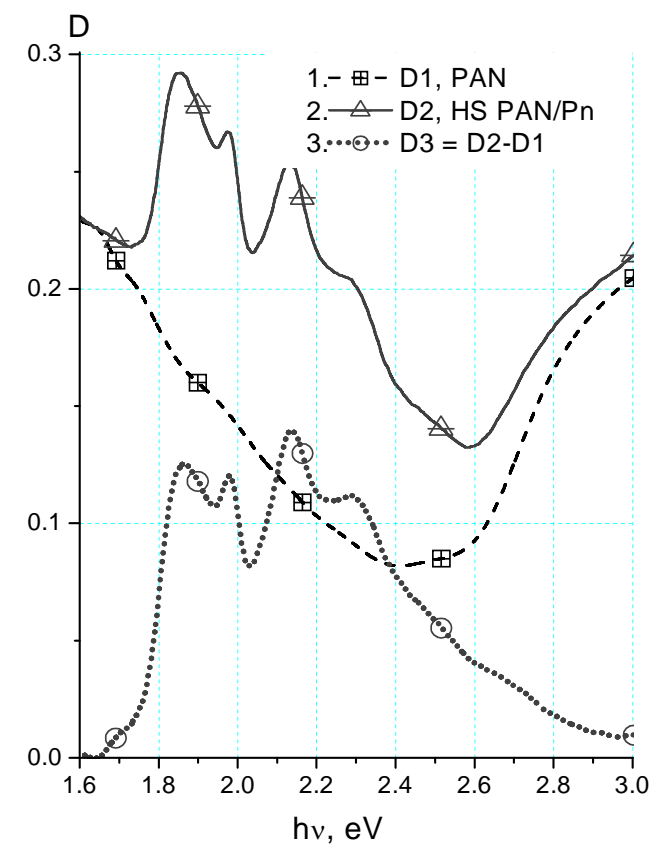

Fig. 3. Optical density spectra $D$ of polyaniline (1), polyaniline/Pn (2) heterostructures (HS) and their difference (3).

\subsection{Photovoltaic properties of polyaniline/OS heterostuctures}

The maximum $\varphi_{s}$ values in the spectra of the PAN/Pn and PAN/MPP heterostructures are approximately the same, i.e., they do not depend explicitly on material or conductivity type of OS. In the whole spectral region, the $\varphi$ values of the heterostructures qualitatively correlate with the absorption spectrum of OS (the more photosensitive component of OS).

The comparison of the photovoltage for PAN/Pn heterostructure with that for reference structures ITO/Pn shows that formation of heterostructure leads to an increase of $\varphi$ (as compared with reference structures) by a factor of $4.1 \pm 0.5$ (Fig. 4). This value is practically independent of the illumination direction but depends on the energy of exciting quanta in this region. The increment of $\varphi$ is maximal in the region of weak absorption in polymeric films $(2.4-3.0 \mathrm{eV})$ and is $30 \%$ less in the region of their strong absorption (1.9-2.3 eV).

The spectra of photovoltage and absorption of the ITO/PAN/MPP heterostructures are well qualitatively correlated with each other (Fig. 5, curve 2,4), which testifies that the rate of recombination of charge carriers at their boundary is low. On the contrary, the spectrum of $\varphi_{c}$ for the reference structure ITO/MPP that is fabricated simultaneously with the above-mentioned heterostructure under the same technological conditions shows the strong anticorrelation at $h v>2.1 \mathrm{eV}$, which testifies to the presence of a great amount of recombination centers for charge-transfer states, especially with the energy $2.7 \mathrm{eV}$, on the free surface of MPP films and the recombination centers in a low concentration for Frenkel excitons that are mainly formed in the interval $2.0-2.3 \mathrm{eV}$ [6]. It is probable that heating the ITO/PAN structures in vacuum up to $370 \mathrm{~K}$ before using the MPP film prevents the creation of the recombination centers for charge-transfer states. As a result of using the MPP film, $\varphi$ of heterostructure increases by a factor from 1.8 to 19 in the region $h v>2.1 \mathrm{eV}$ as compared with that of the ITO/MPP structure.

This essential growth of photovoltage in the heterostructure relatively to that in ITO/OS structures indicates formation of a potential barrier near the boundary PAN/OS with a low concentration of the recombination centers for excitons and charge carriers, i.e., an increase of the internal electric field value. The presence of the internal electric field leads to that the process of photogeneration of charge carriers near the boundary (the space charge region) dominates over their recombination.

\subsection{Photovoltaic properties of the PEDOT-PSS/OS heterostructure}

The deposition of MPP films on a PEDOT-PSS film was performed at the temperature of substrate $370 \mathrm{~K}$, because the photosensitivity of MPP layers is maximal at this temperature of substrates [5]. The measurements of the spectral dependences of photovoltage for the PEDOT-PSS/MPP heterostructure showed that their $\varphi$ value was several times greater than $\varphi_{s}$ value for components, and is maximal in the region of strong absorption in MPP layers (Table, Fig. 6). This indicates that, near the polymer/MPP boundary, there appears an internal electric field that enhances the efficiency of separation of photogenerated carriers.

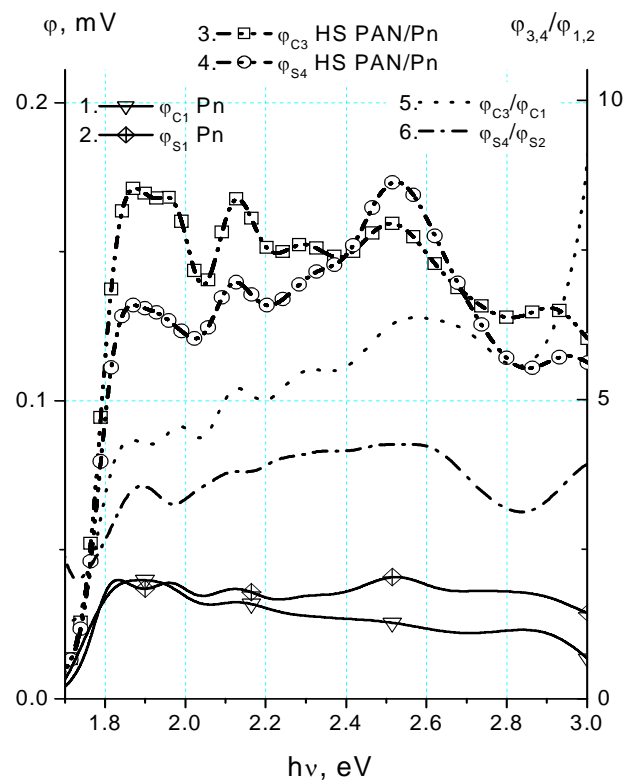

Fig. 4. Photovoltage spectra of $\mathrm{ITO} / \mathrm{Pn}(1,2)$ and polyaniline/Pn $(3,4)$ heterostructures (HS) under illumination through ITO $(1,3)$ and free surface $(2,4)$, as well as spectra of the photovoltage ratio for heterostructures and $\operatorname{ITO} / \operatorname{Pn}(5,6)$. 


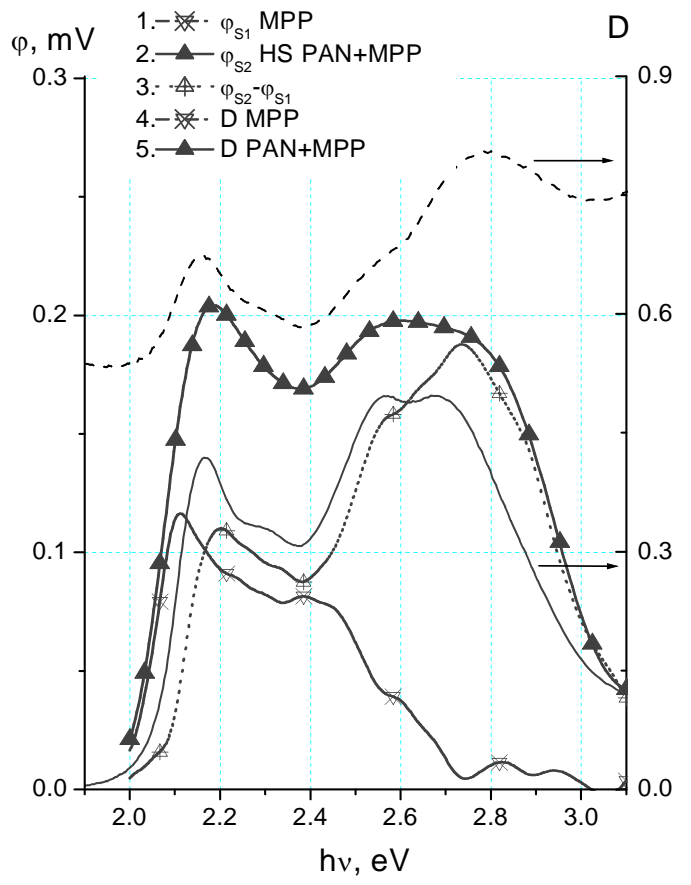

Fig. 5. Photovoltage spectra under illumination of free surface of ITO/MPP (1), polyaniline/MPP (2), and their difference (3), as well as spectra of optical density $D$ for ITO/MPP (4) and polyaniline/MPP (5) heterostructures (HS).

The comparison of the data for the PEDOTPSS/MPP and PAN/MPP heterostructures showed that the ratio of $\varphi$ in the PEDOT-PSS/MPP heterostructure to that in the ITO/MPP one is significantly ( 5 to 12 times) higher than those for the corresponding heterostructures with PAN. This testifies to the formation of a greater potential barrier at the PEDOT-PSS/MPP boundary than that in the PAN/MPP structures, because the optical density of PEDOT-PSS films is less than that of PAN films.

The formation of the PEDOT/Pn heterostructure leads to a greater increase of $\varphi$ relatively to that for Pn films which is independent of the illumination direction (Fig. 6, curves 1 and 2). As a result, the maximal value of $\varphi$ for the PEDOT-PSS/Pn heterostructure in the energy region of 2.0-2.2 eV is 45 times greater than that for the PAN/Pn heterostructure (Fig. 4) and 4 times greater than that for the PEDOT-PSS/MPP structures (Fig. 6). This testifies that the height of the potential barrier near the PEDOT-PSS/Pn boundary is significantly higher than that in the PAN/Pn and PEDOT-PSS/MPP structures.

In the energy interval of $2.6-3.0 \mathrm{eV}$, the ratio of $\varphi_{s}$ for PAN/MPP and ITO/MPP structures as compared to the ratio $\varphi_{s}$ in the energy interval $2.0-2.4 \mathrm{eV}$ is essentially larger, which can be caused by more efficient separation of excitons with charge transfer that makes the main contribution to photogeneration of charge carriers in MPP films in this region [6]. A similar situation is observed for the PAN/MPP structures (Fig. 6).

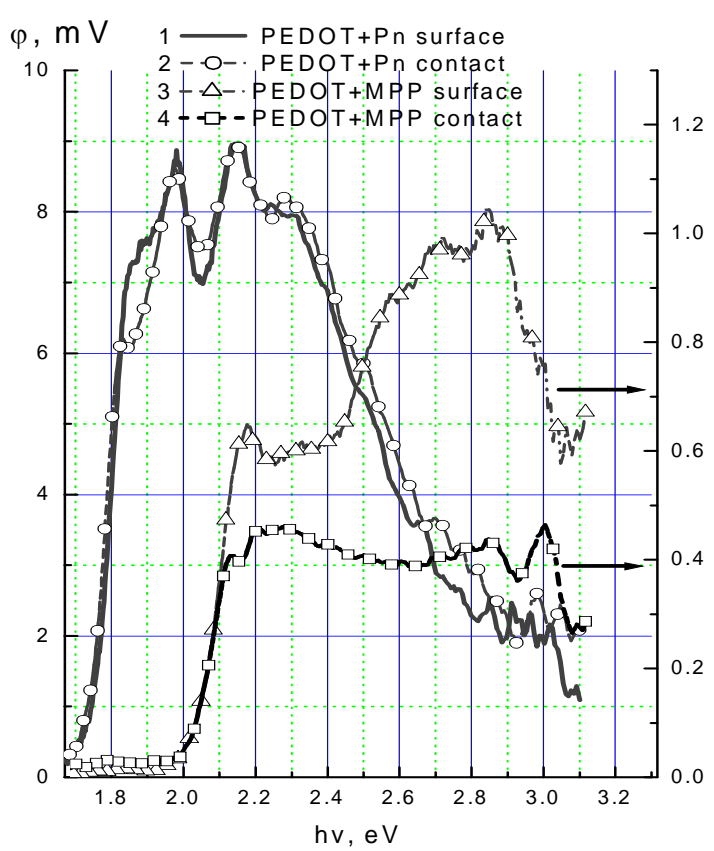

Fig. 6. Photovoltage spectra of heterostructures PEDOTPSS/Pn $(1,2)$ and PEDOT-PSS/MPP $(3,4)$ under illumination of free surface of the films Pn (1), MPP (3), and PEDOT-PSS $(2,4)$.

\section{Conclusions}

It is established that the photovoltage of PAN films deposited onto ITO layers on glass substrates is by 2-3 orders less than that of MPP and Pn layers thermally sprayed on similar substrates under illumination by light-emitting diodes with various quantum energies. The subsequent annealing of PAN layers in vacuum for $3 \mathrm{~h}$ at the temperature $370 \mathrm{~K}$ causes the increase of the surface photovoltage, which testifies to a decrease of the concentration of recombination centers that were formed on the surface due to the adsorption of air molecules.

The photovoltage of polymer/organic semiconductor heterostructures produced at the substrate temperature $370 \mathrm{~K}$ is significantly higher than that of components and is maximal in the region of absorption of organic semiconductor layers and lower in the region of strong absorption of PAN. This indicates that, near the polymer/organic semiconductor boundary, there appears an internal electric field that enhances the efficiency of separation of photogenerated charge carriers, while the photogeneration efficiency for carriers in PAN and PEDOT-PSS layers is insufficient yet. The highest photovoltage and height of barriers are observed in the PEDOT-PSS/Pn structures.

The work was partially financed by the Fund of Fundamental Researches of the Ministry of Education and Science of Ukraine, project F25.4/116. 


\section{References}

1. C.J. Brabec, N. Sariciftci, Solarzellen aus Plastik // Erneuerbare Energien 2, p. 28-34 (1997).

2. J.G. Grote, Ya.I. Vertsimakha, The photosensitive polymeric composites from inorganic and organic semiconductors and prospects of their use // Proc. XIV Intern. Symposium "Advance Display Technologies", October 10-14, 2005, Crimea, Ukraine, p. 216-224.

3. N. Berezina, V. Syromyatnikov, A. Ishchenko, A. Verbitsky, Ya. Vertsimakha, Effect of polymer matrix on photosensitivity of meso-Cl polymethine dye based composites // Functional Materials 13 (4), p. 676-680 (2006).

4. O. Aksimentyeva, L. Monastyrski, V. Savchyn, P. Stakhira, Ya. Vertsimakha, B. Tsiz, Electronic processes in the porous silicon conducting polymer heterostructures // Mol. Cryst. Liquid Cryst. 467, p. 73-83 (2007).

5. O.I. Aksimentyeva, V.M. Beluh, D.O. Poliovyi, V.V. Cherpak, P.Y. Stakhira and D.Y. Volynyuk,
Thermo-vacuum depositon and electrooptical properties of polyaniline thin films // Mol. Cryst. Liquid Cryst. 467, p. 143-154 (2007).

6. Ya. Vertsimakha, P. Lutsyk, K. Palewska, J. Sworakowski, and O. Lytvyn, Influence of optical and photovoltaic properties of thin films of N,N`-dimethyl-3,4,9,10-perylenetetracarboxylic acid diimide // Thin Solid Films 515 (20-21), p. 7950-7957 (2007).

7. Ya.I. Vertsimakha, S.V. Timofeev, V.I. Trofimov, B.R. Tsizh, Photoelectrical properties of heterostructures of cadmium sulphoselenides (CdSSe) and pentacene // Zhurnal prikladnoi spektroskopii 53, p. 685-688 (1990) (in Russian).

8. Ya. Vertsimakha, A. Verbitsky, Oxygen effect on photovoltaic properties in pentacene based barrier structures // Synthetic Metals 109 (1-3), p. 291-294 (2000).

9. Ya.I. Vertsimakha, Photoelectrical properties of interfaces between organic films and CdSSe // Mol. Cryst. Liquid Cryst. 355, p. 275-288 (2001). 